\title{
The impact of non-emergency medical use on the United States health care system: a retrospective study
}

\begin{abstract}
Study Objective: This study analyzes the 2010 NEDS data set to investigate and distinguish the characteristics of non-emergency visits compared to emergency visits. Additionally, this study uses five different statistical methods of analysis to identify the percentage of emergency visits made for non-emergency conditions, determine the impact of non-emergency medical use on patient outcomes of inpatient mortality, emergency department waiting time and total emergency department charges.
\end{abstract}

Methods: In this study various methods of data analysis of the 2010 NEDS data set were used such as: descriptive statistics, EDCPT severity level, NYUED classification algorithm, variance and logistic regression.

Results: Results of descriptive statistics show that between 54.02 to 82.7 percent of all emergency department visits were made for conditions found to be routine, lowseverity, or non-emergent. Results of analysis of variance show significant statistical differences between the means of non-emergency visits and emergency visits. Finally, results of logistic regression suggest that there are statistically significant predictive relations between patients' demographic characteristics and outcomes of emergency visits in $76.5 \%$ of all cases.

Conclusion: The results of this study lead to the conclusions that a significant number of emergency department visits are made for non-emergency conditions, which can be depicted as the main basis for non-emergency medical use as to negatively impact patient outcomes of inpatient mortality, emergency department waiting time and total emergency department charges.

Keywords: non-emergency, ED rooms, EMTAL, NEDS data, clinical analytics, statistical modeling, CPT1-CPT15, algorithm analysis, NYUED algorithm, logistic regression analysis, ED visits, emergency
Volume 3 Issue 2 - 2015

\author{
Patrick Casimir \\ Department of Master Science of Health Informatics, \\ Jacksonville University, USA
}

Correspondence: Patrick Casimir, Department of Master Science of Health Informatics, Brooks Rehabilitation College of Health Sciences, Jacksonville University, Jacksonville, FL 3221।, USA,Tel 9546I4I I78,Email pcasimi@ju.edu

Received: August 10,2015 | Published: November 3, 2015
Abbreviations: ED, emergency department; EMS, emergency medical screening; CMS, centers for medicare and medicaid services; NEDS, national emergency department sample; HUCUP, health care cost and utilization project; AHRQ, agency for health care research \& quality; CSV, comma separated value; DUA, data use agreement; SEDD, state emergency department database; SID, state in patient databases; CPT, current procedural terminology; LOS, length of stay; DRG, diagnosis related group; POA, presence of admission; ANOVA, analysis of variance; ROC, receiver operating characteristic; IOM, institute of medicine's; ACEP, American college of emergency physicians

\section{Introduction}

The delivery of emergency medical services in the United States has been a controversial and troubling issue for more than three decades. Consequently, non-emergency medical use, regarded as the utilization of emergency medical services for conditions that are considered non-emergent, has grown rapidly and continues to be an alarming issue for health authorities, private and public hospitals and a much debated and studied subject by researchers and experts in the field. Although emergency department rooms are primarily designed to provide urgent, critical and emergent medical care services, millions of Americans use emergency department (ED) rooms to seek treatment for non-emergency medical conditions as they successfully by pass the need to visit a primary care physician by using ED rooms as alternatives to primary medical care providers. Throughout this study, non-emergency medical use will refer to the common practice of neither receiving emergency medical services for health conditions that are neither emergent nor life threatening. Predominantly, such utilization results in a non-emergency visit, a visit for which treatment is not critically urgent, not lifesaving and not required within less than 60minutes (42U.S.C.1395dd, 1986). In today's literature, various terms have been used to describe nonemergency visits. Non-emergency visits are often labeled as nonurgent, avoidable, or preventable. While all those terms do not have a precisely similar definition, they all bear the common understanding that such visits were not truly emergent and that they could have been handled elsewhere such as in a primary care setting or in a urgent care center.

On April 7, 1986 President Ronald Reagan signed EMTAL Aintolaw. EMTAL Amandates hospitals to performa emergency medical screening (EMS), determine if an emergency medical condition (EMC) exists, stabilize the EMC if possible and/or transfer and accept EMC's transfer for all patients who come to emergency 
rooms to seek treatment regardless of patients' ability to pay for those emergency care services received. The primary intent of EMTALA was to guarantee emergency medical services for all ED patients and to stop hospitals from the usual practice of dumping patients because of their in ability to pay or lack of health insurance coverage. However since the passage of EMTALA, utilization of ED rooms has considerably increased, which has created a lot of controversies leading many to blame EMTALA for the rise in emergency medical use. Despite EMTALA's revisions in 2003 by the Centers for Medicare and Medicaid Services (CMS) which were intended to clarify and simplify its obligations and limitations, the utilization of ED rooms for both emergency and non-emergency conditions has continued to increase beyond capacity.

\section{Case presentation}

This retrospective study is designed to analyze the 2010 National Emergency Department Sample (NEDS) dataset of the Health care Cost and Utilization Project (HCUP) to investigate and distinguish the characteristics of non-emergency visits, estimate the number of ED visits made for non-emergency conditions, determine the impact of non-emergency medical use on patient outcomes of mortality, ED waiting time and total ED charges and make viable recommendations that can help unravel the ongoing and increasing problem of nonemergency medical use. The 2010 NEDS data set was used for this retrospective study because it was the most recent and latest data set available from HCUP when this study was initiated. Analyses and interpretations will be made from a number of sources including business intelligence, clinical analytics and statistical modeling tools. Specifically, descriptive and inferential statistical analyses will be performed on the HCUP's 2010 NEDS data set to find differences among ED visits in order to determine whether those ED visits were due to emergency or non-emergency conditions.

The 2010 NEDS is a data set made of a sampling population of over 28million records of ED visits collected at 961 hospitals across the nation and stratified at almost $20 \%$ of hospital-based ED visits, which makes this dissertation an empirical research since no other previous known studies have been conducted to use the 2010 NEDS data set to investigate ED utilization for non-emergency conditions (HCUP, 2010). Because of the enormous size of the 2010 NEDS data set, data records from previous years and/or other data sources will not be used in this dissertation. This study will investigate the impact of non-emergency medical on the health care system in the United States. Although previous studies have researched the utilization of ED medical services at specific hospitals, states, regions and for particular diagnoses no study has been done to investigate and determine the negative impact of non-emergency medical use on outcomes of ED waiting time, ED cost per visit and in patient mortality. ${ }^{1-4}$

\section{Data set and methods of data analysis}

The 2010 national emergency department sampled at a set, used for this dissertation, is a compilation of emergency department visits records that derive from the HCUPNEDS data base. The 2010 NEDS data set was purchased from the Agency for Health care Research \& Quality (AHRQ) for a discounted price for students and received in a DVD disk with 4 files made of compressed data in comma separated value (CSV) format. It is important to note that users of the 2010 NEDS data must be aware of and comply with the data use limitations, which requires all users to complete the Data Use Agreement (DUA) training, sign and return a copy of the DUA to the Agency for Health care Research \& Quality (AHRQ) Under Federal law, violators of the DUA can be fined upto $\$ 10,000$ and imprisoned for up to 5 years. The 2010 NEDS contains ED visits records originated from the State Emergency Department Database (SEDD) and the State In patient Databases (SID) collected across 961 hospitals nationwide during that same calendar year. According to HCUP, "the NEDS are the largest all-payer emergency department (ED) data base in the United States, yielding national estimates of hospital-based ED visits. Unweighted, it contains data from approximately 29 million discharge search year. Weighted, it estimates roughly 130million ED visits." ${ }^{15}$ The 2010 NEDS data set consists of four main files structured a stables: the NEDS Core File, the NEDS Supplemental ED File, the NEDS Supplemental In patient File and the NEDS Hospital Weights File. The 2010 NEDS Core File is the largest of the four files and includes $28,584,301$ million ED visits records or $100 \%$ of the sampling population whether they resulted inpatient's admission to a hospital or not. The 2010 NEDS Core File contains over 40 data elements.

The 2010 NEDS Supplemental ED File includes 24,192,665million records of ED visits in which patients were not directly admitted to the hospital. The 2010 NEDS Supplemental ED File mainly contains procedural data elements such as CPT1 for Current Procedural Terminology (CPT) and PR_ED1 for ICD-9-CM procedures per formed during ED visits. The 2010 NEDS Supplemental In patient File includes 4,391,636million records of ED visits that resulted in admission to the same hospital. The 2010 NEDS Supplemental Inpatient File contains data elements such as length of stay (LOS) during inpatient stay, total charges during inpatient stay, discharge information during inpatient stay and Diagnosis Related Group (DRG) used on discharge date and calculated without presence of admission (POA). Finally, the 2010 NEDS Hospital Weights File includes 961 records of hospitals used for the collection of ED visits. The 2010 NEDS Hospital Weights File contains data elements such as number of ED visits per hospital, hospital's ownership and governance, hospital's region, hospital's trauma level designation, hospital's teaching status and hospital's urban-rural designation. All four files comprise the unique key identifiers HOSP_ED and KEY_ED that can be used to perform record linkage and cross join among the four main files or tables.

\section{Descriptive statistical analysis}

In this study, descriptive statistical analysis was used to produce a well detailed summary and makeup of the 2010 NEDS Core file in terms of frequencies and percentage counts and uncover general trends and variations among different groups and categories of ED visits. First, data elements were statistically analyzed with the emphasis to determine the frequency and percentage of ED visits per individual statistical category or grouping such as injury severity, income, death in the ED, chronicity, quarter of discharge, age, region, presence of injury, disposition from the $\mathrm{ED}$, type of injury, gender, location of residency, month, day, ED charges, intent of injury, payer and the type of ED event using basic SAS functions such as PROCSORT, PROCSQL, PROCMEANS, PROCTABULATE, PROCUNIVARIATE, PROCFREQ. Second, extensive descriptive statistical analysis was carried out to compare ED visits per payer group, region, age group, income group, gender and location of residence. For each of those groupings, ED visits were compared and analyzed across the type of ED event, presence of injury, chronicity, number of injury and severity of injury. Such analyses were necessary to illustrate variations among sub-categorical variables. 
As an example, those analyses made it possible to differentiate the types of ED events per sub-categories of payer, age, region, gender, income and location. Payer, age, region, gender, income, location were divided in various sub-categories. Each of those sub-categories was analyzed across types of ED events in terms of routine visit or admission, presence of injury, number of injury, severity of injury and chronicity of conditions.

\section{ED CPT severity level analysis}

In this study, CPT severity level analysis was applied to the 2010 NEDS with the intent to establish the usefulness of this method in investigating ED visits of non-emergency and emergency attributes. In this study, data elements CPT1-CPT15 are statistically analyzed and grouped in two categories of non-emergency and emergency visits. Unlike the traditional classification analysis, in which ED services with CPT coding 9928 3are defined as of high acuity and severity, services with CPT coding of 99283 are analyzed and grouped depending on the association with or the presence of injury. ED services with CPT coding 99283 without the presence of injury are classified as non-emergent and ED services with CPT coding 99283 with the presence of an injury diagnosis are classified as emergent. Because the data elements CPT1-CPT15 and INJURY are part of two different data files, it was necessary to link the2010 NEDS Core File to the 2010 NEDS Supplemental ED File to make it possible to analyze CPT codes with the diagnosis of injury. The decision to reclassify various 99283 CPT codes was based on the understanding that many of the symptoms attributed to 99283 CPT codes can be of minor severity and complexity unless if they are associated with some types of injuries. By example, symptoms such as eye pain, fever, headache, milddyspnea, abdominal pain and cellulite is all require treatments and procedures that can be handled effectively and safely either at urgent care center so physician's offices. While some conditions linked to 99283 CPT codes can require emergency medical services, others do lack the level of severity and urgency needed to be accepted as emergency conditions.

\section{NYUED classification algorithm analysis}

The NYUED Algorithm combined with the SAS software tool available atthe NYU website were downloaded and used to analyze the 2010 NEDS data set (NYU Wagner, 2014). Furthermore, due to issues linked to the 2010 NEDS data set and the fact that the NYUED algorithm was designed to work with SAS7 or 8, modifications were made so that the NYUED algorithm can be used with SAS 9.3 Prior to performing the NYUED algorithm analysis, DX1, the data element for the principal diagnosis, was cleaned of all data records that were either missing or valued a sinvlandinen (for in valid and inconclusive). A total of 8881 amongst 28,584,301 the ED records were excluded from the analysis. Within DX1 of 2010 NEDS, a total of 10439 different primary diagnosis codes were used for the NYUED algorithm analysis. Although we will use the same classification schemes, our analysis will only consider two main categories of ED visits, which makes this method of analysis very complex and timeconsuming. In one hand, ED visits, with in groups 1-2, classified as non-emergent and emergent primary are treatable will be considered as non-emergency visits. In another hand, ED visits, with in groups $3-4$, classified as emergent with ED care needed and preventable/ avoidable and emergent with ED care needed and not preventable/ avoidable will be considered as emergency visits. The basis for such an analysis to classify ED visits depends on whether emergency medical care services were emergent or non-emergent at the time of the ED visit, instead of consideration that the condition that led to the ED visit was either avoidable or preventable.

\section{Analysis of variance}

In this study, the utilization of ANOVA Single Factor from Excel 2007 will be necessary to test statistically significantly difference between non-emergency visits and emergency visits by comparing differences in the means of numbers of ED visits that resulted in admission and those that did not, ED visits associated with injury and ED visits not associated within jury, ED visits with one injury or less and those with multiple injuries, ED visits for high severity injury and those for lows verity in jury and ED visits for chronic conditions and those for non-chronic conditions. The goal of using ANOV At estimate differences among scores of ED visits within the 2010 NEDS data set is to test the hypothesis that those five critical criteria of admission, presence of injury, sever it you injury, number of injuries and chronic it can be indicators of whether ED visits are made for emergency or non-emergency conditions. Infact, the ANOVA analysis will help us test the under lying probability that ED visits recorded as routine, nonjury, low injury severity, non-chronic and with one injury or less are more likely to result in non-emergency visits.

To do the analysis of variance, first, aggregated at afro descriptive analysis were used to design five groups of data sets based on the criteria previously mentioned. Second, the SAS tool was used to perform as in glefactor ANOVA. Each group of data set is made of 2 sets of data to be compared by ANOVA based on the format shown in below. For each analysis, when the value of Fraction is greater than $\mathrm{F}$ crit, the under lying hypothesis will be confirmed. Yet, if the value of Fraction is smaller than the F crit, which will indicate that the underlying hypothesis can only be explained by chance.

\section{Logistic regression analysis}

In this study, logistic regression analysis will be used to test if the rear statistically significant relations between patient's demographic characteristics and outcomes of emergency visits. Logistic regression analysis will be useful in predicting the probability that outcomes of ED visits, represented by the newly created data element emergency a dichotomous depend end variable with value of 1 for an emergency visit and value of 0 for an on-emergency visit, are in flounced by as in gleor multiple in depend enter predictor variables such as age, gender, injury, income, payer type and location of residence. The dichotomous variable emergency was created from there categorization of ED event variable. By default, the SAS software models the probability that the outcome variable equals 0 . In this analysis, we will model the probability that the response or outcome emergency equals 1 by adding the option "descending" to all logistic regression analysis coding. Our analysis will model appositive response variable that predicts the odds ratio that an emergency visit occurs. No additional steps were taken to clean the data from missing values because the SAS application performs such deletions automatically.

In this study, logistic regression analysis, in its simple terms, will be used to test or investigate the likelihood of an emergency visit as a function of one or multiple predictors. Simple logistic regression will be used to investigate the relation between one binomial outcome and one predictor or in dependent variable. Multiple logistic regressions will be used to investigate the relation between one binomial outcome and multiple predictors or in dependent variables. Consequently, multiple regression analysis allows in vestigators to assess how the 
relationship between the outcome variable and in depends invariable is influenced by the addition of one or multiple independent variables. As an example, age can be added to as impleregression analysis in which gender was in itially used as independent variable to predict the likelihood of an emergency visit. In doing so, it is now possible to determine whether age has affected the association between gender and the outcome either positively or negatively.

\section{Results}

\section{Results of descriptive statistical analysis}

Over whelmingly, results of descriptive statistical analysis of the 2010 NEDS provided significant numerical observations with in the 2010 NEDS data set that confirmed assumptions indicating that a considerable number of ED visits are made for non-emergency events (Figure 1). Such numerical observations show that $82.78 \%$ of ED visits were routine whilst $16.91 \%$ resulted in admission, $76.72 \%$ of ED visits were not injury related whilst $23.28 \%$ had an injury diagnosis, $83.06 \%$ of ED visits were for non-chronic conditions whilst $16.91 \%$ were not, $98.63 \%$ of ED visits were for non-severe injuries whilst $1.04 \%$ was not and mean of ED charges for routine ED visits were $\$ 2128$ whilst mean of ED charges for ED visits that resulted in admission were $\$ 1593$. These results are a clear indication that a significant percentage of ED visits were made for non-emergency events. Even though not all routine ED visits are made for non-emergency conditions, a substantial amount of routine ED visits are driven by non-emergency events. Although the use of descriptive statistical analysis of the 2010 NEDS data set does not permit to calculate the number nor the percentage of ED visits made for non-emergency conditions, further statistical methods of EDCPT Severity level analysis and NYUED Algorithm classification analysis will provide us with better numerical estimations of such values.

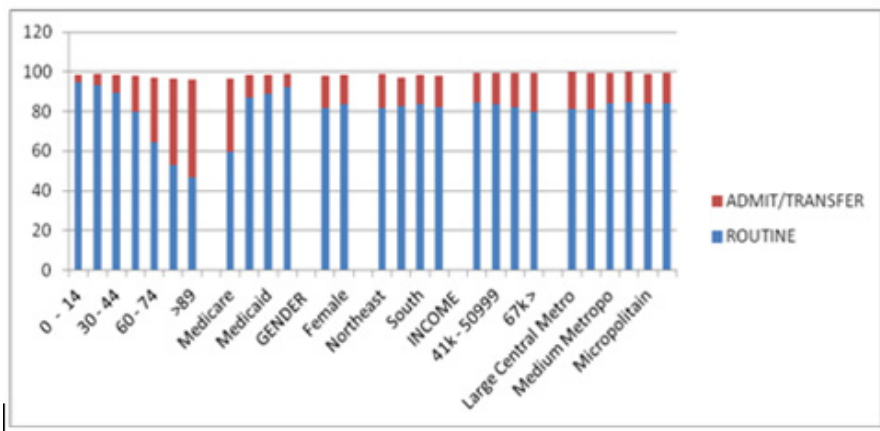

Figure I Percentages of ED visits per snsfer across age, payer, gender, region, income and location groups.

\section{Results of EDCPT severity level analysis}

Evidently, results of EDCPT severity level analysis of the 2010 NEDS showed that there are statistically effective procedural methods that can be used to differentiate non-emergency visits from emergency visits. Those procedural methods show that $54.02 \%$ of ED visits were of low severity or made for non-emergency conditions whilst $45.98 \%$ were of high severity or made for emergency conditions (Figure 2).

\section{Results of NYUED classification algorithm analysis}

Accordingly, the results of NYUED classification algorithm analysis of the 2010 NEDS validate our hypotheses that there exist diagnostic methods to differentiate non-emergency visits from emergency visits. As a diagnostic based statistical method of analysis, the NYUED algorithm's results show that $65.78 \%$ of ED visits were for non-emergency reasons whilst $34.22 \%$ were emergencyreasons (Figure 3).

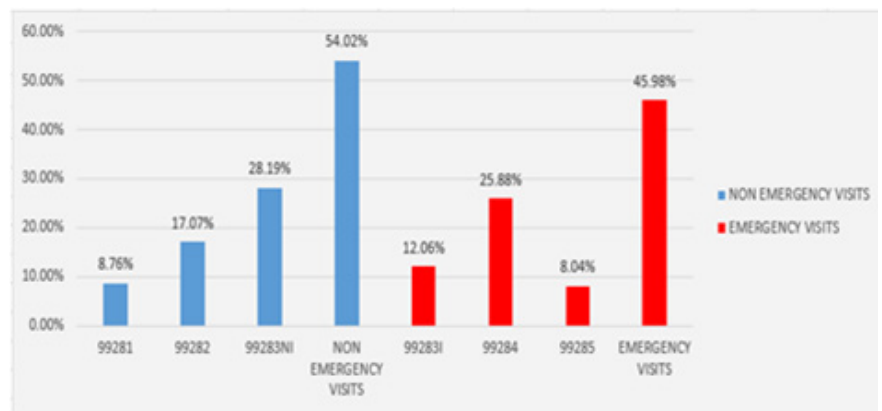

Figure 2 Distribution of percentages of CPT codes in the 2010 NEDS associated with presence of injury.

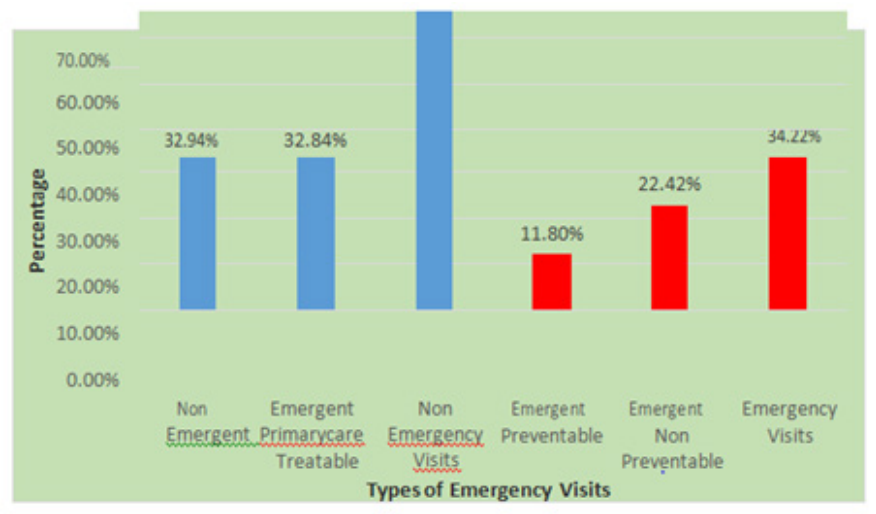

Figure 3 Final re classification of percentages of ED visits of the 2010 NEDS per emergency and non-emergency.

\section{Results of analysis of variance (ANOVA)}

Results of analysis of variance with ANOVAs Single Factor from Excel 2007 confirmed differences in means of variables Edevent, Injury, Injury_Severity, Multinjury and Chron1 through F ratio values of $46.96,21.15,88.53,53.84$ and 80.08 significantly greater than the value of $\mathrm{F}$ crit value of 4.03. Those values indicated that ED visits, associated to conditions that were routine, without injury, of low severity injury, not chronic and the absence of multiple injuries, are made for non-emergency reasons compared to ED visits in which patients were either admitted, had injury, suffered a high severity injury, had a chronic condition and diagnosed with multiple injuries. In terms, those results demonstrated that ED visits made for emergency conditions are statistically significantly different of those made for non-emergency conditions because of differences between the means of the ED visits that were routine vs admit, ED visits with no injury vs injury, ED visits with low injury severity vs high severity, ED visits that were not chronic vs chronic and ED visits with one injury or less vs multiple injuries.

\section{Results of logistic regression analysis}

Lastly, results of a simple logistic regression analysis (CI=95\%; $\mathrm{OR}=1.851$ ) showed predictive associations between age and outcomes of ED visits represented by the dependent variable EMERGENCY. Also, results of a multiple logistic model (CI=95\%; $\mathrm{OR}=0.8,0.78,1.02,0.92,1.72,1.01)$ depicted relations between certain 
patient's demographics and characteristics represented by independent variables of AGE, FEMALE, PL_NCHS 2006, ZIPINC_QRTL, PAY1 and outcomes of ED visits. As illustrated in Figure 4, both models were validated by receiver operating characteristic (ROC) curves that illustrated the fitness and performance of both models at predicting how outcomes of ED visits can be influenced by dependent factors. ROC graphical plots exemplified that both models correctly predicted outcomes of ED visits for $76.9 \%$ of occurrences.

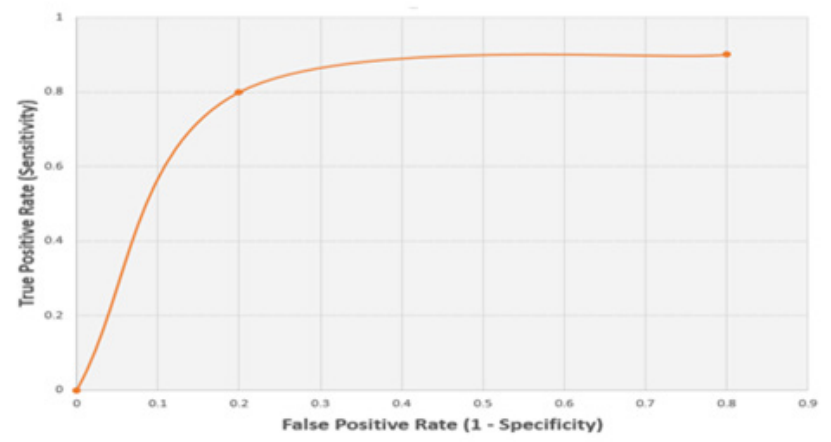

Figure 4 ROC curve for the multiple logistic regression models with an area under the curve of 0.769 .

\section{Discussion and conclusion}

Clearly, over the last three decades, non-emergency medical use has grown at an alarming rate, which has also impacted the health care system in the United States in negative ways. ${ }^{6,7}$ So far, study results of statistical analyses provided consistent insights that have allowed us to uncover that a significant amount of emergency visits in 2010 were made for non-emergency conditions. The focus of our discussions is to show that non-emergency medical use can have a negative impact on the delivery of emergency care services and the health care practice in general by negatively impacting patient outcomes of ED waiting time, total ED charges and inpatient mortality.

First, Non-emergency medical use can play a significantly negative role in the increase of the patient outcome of ED waiting time. Like so, non-emergency medical use is commonly associated with lengthy ED waiting time by increasing the work loads of ED staff and putting additional strains on other resources. While hospital's ED usually prioritize ED patients based on acuity, which allows the treatment of critically injured and trauma patient first, time and resources must still be invested in the triage and emergency medical screening of all people present at the ED as legally mandated by EMTALA. In general, most hospitals lack resources and enough employees to handle all patients who come to their EDs with urgent and emergency conditions. Nevertheless, hospitals struggle to medically screen patients with conditions deemed of non-emergency. Similarly, the Institute of Medicine's (IOM) Committee on the Future of Emergency Care wrote, "when the ED is at full capacity, treating additional patients who could be cared for in a different environment means fewer resources-physicians, nurses, ancillary personnel, equipment and time and space available to respond to emergency case". ${ }^{8}$

As shown in Figure 5, Figure 6 the number of non-emergency visits between 2000 and ED waiting times between during the same period display a somehow similar upward trend. Between 2000 and 2009 , the number of non-emergency visits increased from 61.76 to 76.19 million, an increase of $23 \%$, whilst ED waiting times increased from 45 to 58.1 minutes, an increase of $29 \%$. Second, non-emergency medical use frequently manifested through ED overcrowding has been known to be factor in the surge of the secondary patient outcome of ED charges in recent years. ${ }^{7}$ Indeed, it is important to recall that EMTALA's man date requires hospital's EDs to conduct an emergency medical screening on all ED patients regardless of their ability to pay for care services rendered. Because hospitals do not receive federal incentives to counter balance for those financial losses, EMTALA's legal and regulatory requirement has been blamed for causing hospitals remendous financial losses and forcing them to continuously raise ED charges to offset for uncompensated care. Un questionably, some studies have designated non-emergency medical use and/or related in appropriate use of ED care as major causes of mounting ED charges. ${ }^{9}$ Accordingly, IOM Committee on the Future of Emergency Care declared, "But uncompensated care can be an extreme burden at hospitals that have large numbers of uninsured patients. Many hospital ED and trauma enter closures are attributed to financial losses associated with emergency and trauma care". ${ }^{8}$ In the same light, in are port published in 2006 it was suggested, "primary care received in the ED is sometimes viewed as source of excess cost, since hospital charges include mark-upto cover a variety of over head expenses". ${ }^{10}$ Third, non-emergency medical use has been shown to be connected to the patient outcome of inpatient mortality.

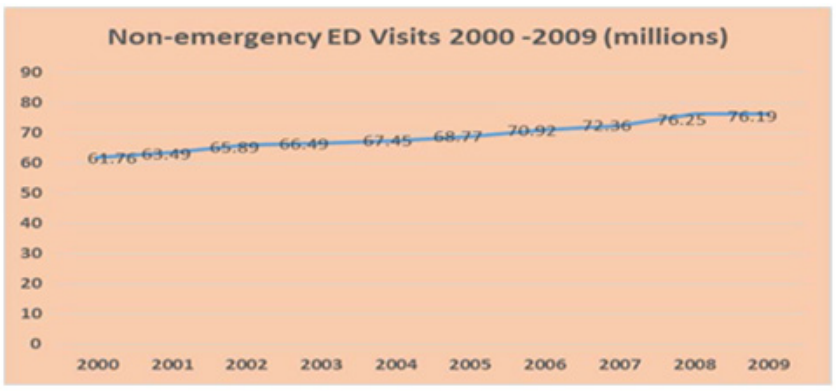

Figure 5 Non-emergency visits $(2000-2009)$.

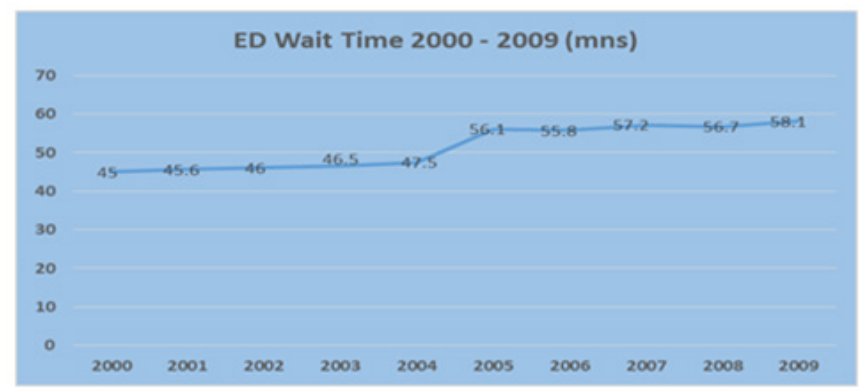

Figure 6 ED waiting times $(2000$ - 2009).

Granting the 2010 NEDS data set does not contain a data element for in patient mortality, our interpretations and findings from various studies will suffice to demonstrate how non-emergency medical use can impact mortality of patients following their admission as in patients. Within the health care spectrum, increases in inpatient mortality has generally been linked to common diseases and health risks of cancer, congestive heart failure, epidemic outbreaks, medication errors, medical negligence, obesity, diabetes, smoking, excessive alcohol drinking, substance abuse and other injuries that derived from cuts, drowning, falls, fires, firearms, machineries, motor vehicles, natural disasters, poisons, struck and suffocations. 
Mostly, general assumptions only consider simplistic factors, those easily understood and identified, as causes of inpatient mortality. Nevertheless, recent studies have found consequential relations between ED overcrowding and the increase of inpatient mortality. Thereby, a 2012 study of 995, 379 ED across 187 hospitals, found that ED overcrowding can increase inpatient mortality by oddsof $5 \%$. Subsequently, the authors wrote "patients who were admitted on days with high ED crowding experienced $5 \%$ greater odd so in patient death". In that same observational study, it was shown that odds of inpatient mortality can go as high as $9 \%$ when models were adjusted to simulate ED overcrowding over a period of 3days. Moreover, a 2011 literature review study of 276 articles examining the impact of ED overcrowding on inpatient mortality, stated, eight studies examined the association between ED crowding and mortality. Although ED crowding was measured differently in each study, the majority of these studies found that correlations exist between ED crowding and increased mortality. ${ }^{6}$

In the same perspective, a 2013 study found a positive relationship between ED crowding and inpatient mortality and admitted, "Notably, studies found that ED crowding is associated with higher rates of inpatient mortality among those admitted to the hospital from the ED and discharged from the ED to home". ${ }^{7}$ Lastly, in are port on the solutions to ED crowding, the American College of Emergency Physicians (ACEP) provided a critical in sight describing the association between ED crowding and inpatient mortality. ${ }^{11}$ As suggested earlier, there is increasing evidence confirmed by the results of this study and findings from various other studies to assert the negative impact of non-emergency medical use on patient outcomes of ED waiting time, ED cost per visit and inpatient mortality. In conclusion, this study has shown that in 2010 a significant majority of ED visits were caused by medical events deemed non-emergent, which caused ED patients to wait longer for emergency medical services, pay more for ED care and die at a higher rate.

\section{Acknowledgements}

None.

\section{Conflict of interest}

The author declares no conflict of interest.

\section{References}

1. Delia D, Cantor J. Emergency department utilization and capacity. Robert wood Johnson foundation research synthesis report No. 17; 2009.

2. Division of Health Care Finance and Policy (DHCFP). Preventable/ avoidable emergency department use in Massachusetts fiscal years 2004 to $2008 ; 2010$.

3. Cunningham P. Nonurgent Use of Hospital Emergency Departments. Center for studying health system change website; 2011.

4. Ramgoolam A, Green JJ, Betz R, et al. Emergency Department Use for Non-Urgent Care: Patient Perspectives and Possible Solutions. The Children's Health Fund Website; 2008.

5. Nationwide emergency department sample (NEDS). Healthcare Cost and Utilization Project (HCUP). Agency for Healthcare Research and Quality Website; 2010.

6. Johnson KD, Winkelman C. The effect of emergency department crowding on patient outcomes: a literature review. Adv Emerg Nurs J. 2011;33(1): 39-54.

7. Carter EJ, Pouch SM, Larson EL. The Relationship between Emergency Department Crowding and Patient Outcomes: A Systematic Review. $J$ Nur Scholarsh. 2014;46(2): 106-115.

8. Institute of Medicine (IOM). Committee on the Future of Emergency Care in the United States Health System. Hospital-Based Emergency Care. At the Breaking Point. Washington DC, USA: The National Academies Press; 2017.

9. Hsia RY, Kellermann AL, Shen YC. Factors Associated With Closures of Emergency Departments in the United States. JAMA. 2011;305(19):1978-1985.

10. Delia D. Potentially Avoidable Use of Hospital Emergency Departments in New Jersey. Rutgers center for state health policy (CSHP) Website; 2006.

11. The American College of Emergency Physicians (ACEP). Emergency Department Crowding. High-Impact Solutions; 2008. 